\title{
Comparative study of metamodelling techniques in building energy simulation: guidelines for practitioners
}

\author{
Liesje Van Gelder ${ }^{\mathrm{a}, *}$, Payel Das ${ }^{\mathrm{b}}$, Hans Janssen ${ }^{\mathrm{a}}$, Staf Roels ${ }^{\mathrm{a}}$ \\ ${ }^{a}$ Building Physics Section, Department of Civil Engineering, KU Leuven, Kasteelpark Arenberg 40 Bus 2447, 3001 Heverlee, \\ Belgium \\ ${ }^{b}$ The Bartlett School of Graduate Studies, University College London, Central House, 14 Upper Woburn Place, London \\ WC1H ONN, UK
}

\begin{abstract}
Computer simulation of real system behaviour is increasingly used in research and development. As simulation models become more reliable, they also often become more complex to capture the progressive complexity of the real system. Calculation time can be a limiting factor for using simulation models in optimisation studies, for example, which generally require multiple simulations. Instead of using these timeconsuming simulation models, the use of metamodels can be considered. A metamodel approximates the original simulation model with high confidence via a simplified mathematical model. A series of simulations then only takes a fraction of the original simulation time, hence allowing significant computational savings.

In this paper, a strategy that is both reliable and time-efficient is provided in order to guide users in their metamodelling problems. Furthermore, polynomial regression (PR), multivariate adaptive regression splines (MARS), kriging (KR), radial basis function networks (RBF), and neural networks (NN) are compared on a building energy simulation problem. We find that for the outputs of this example and based on Root Mean Squared Error (RMSE), coefficient of determination $\left(\mathrm{R}^{2}\right)$, and Maximal Absolute Error (MAE), KR and NN are the overall best techniques. Although MARS perform slightly worse than KR and NN, it is preferred because of its simplicity. For different applications, other techniques might be optimal.
\end{abstract}

Keywords:

metamodelling strategy, polynomial regression, multivariate adaptive regression splines, kriging, radial basis function network, neural network

\section{Introduction}

Computational models are commonly used in various fields such as engineering and economics to simulate system behaviour. Due to the increasing reliability of these models, simulation is usually more useful and less expensive than real-time experiments, which may not be feasible. Depending on the model complexity, simulations can take from only a second to several days, weeks, or even months. Despite the huge potential of these simulations, excessive calculation time might be a limiting factor, especially in optimisation problems. Moreover, lowering calculation time by reducing the model complexity is not an option as this might lead to less reliable results. To counter this computational barrier, metamodels - also known as surrogate models - have been introduced to replace potentially time-consuming models $[1,2]$. Metamodels aim at mimicking the original complex simulation model via a simplified mathematical model, statistically determined based on original model realisations. The simulation then only takes a fraction of the original simulation time, allowing significant computational savings, without compromising the reliability. Both stationary and dynamic metamodels can be constructed, however the focus of this paper is limited to the former.

\footnotetext{
*Corresponding author: Tel:+32 16321344 Fax:+32 16321980

Email address: liesje.vangelder@bwk.kuleuven.be (Liesje Van Gelder)
} 
Several metamodelling techniques were developed to address this increasing interest, and different applications appeared in the literature during the last few decades. Based on both mathematical and engineering test problems with a varying number of inputs, number of samples, degree of non-linearity, noisy behaviour, and applied fitting algorithm, several metamodelling techniques were compared [1, 3-8]. Amongst others, mainly polynomial regression, multivariate adaptive splines, kriging, radial basis function networks, and neural networks were explored. In the above-mentioned literature, polynomial regression is considered as the worst performing technique, while neural networks are advised for problems with many inputs, and kriging is recommended for highly non-linear problems. When the considered problem is noisy however, kriging typically performs the worst. Depending on the number of samples, the degree of non-linearity and noisy behaviour of the particular problem and also the employed algorithm, yet other techniques may provide better models.

Instead of focussing on the theory of metamodelling and developing new techniques and algorithms, this paper aims at guiding users in metamodelling more complex engineering problems based on readily available algorithms. This was already intended by Simpson et al. [1] and Wang and Shan [2] for design optimisation, however no concrete guidelines were presented. This paper therefore wants to take this one step further by proposing a practical metamodelling strategy. To illustrate this, a building energy simulation example is chosen as metamodelling was only very recently introduced in this field to overcome the time barrier in optimisation $[9,10]$. The time-efficiency of metamodels enable them to also be used in robust optimisation such as in [11] and [12], which generally requires significantly more simulations. Although the metamodels themselves are computationally inexpensive to run, they are not always constructed in the most time-efficient way. Eisenhower et al. [9] and Ferreira et al. [10], for example, both used about 5000 samples to fit their metamodels. As this is not feasible for very computationally-expensive simulation models, this paper proposes a metamodelling strategy dealing with both time-efficiency and reliability: a well-performing metamodel trained on as few samples as possible will be preferred.

Section 2 first describes the theory concerning fitting and validating metamodels. Five groups of metamodelling techniques are described, with a focus on the selection of algorithms: polynomial regression, multivariate adaptive regression splines, kriging, radial basis function networks, and neural networks. Section 3 then proposes a metamodelling strategy, using as little data as possible without compromising the reliability of the metamodel. The building energy simulation application is described in section 4 , and the results for this are presented in section 5 .

\section{Metamodelling theory}

Wang and Shan [2] and Kleijnen and Sargent [13] emphasise the importance of both fitting and validating metamodels in view of model reliability. This is indeed one of the major concerns as metamodelling aims to replace a model without becoming unreliable. Therefore, this section first describes the fitting of a metamodel followed by the validation. Furthermore, a selection of five metamodelling techniques is described as these are thought to be most useful based on literature: polynomial regression (PR), multivariate adaptive regression splines (MARS), kriging (KR), radial basis function networks (RBF), and sigmoidal neural networks (NN).

\subsection{Fitting}

A metamodel is a mathematical function for which the coefficients are determined based on a limited number of input/output combinations. To create $n$ samples of the $p$ inputs, a Monte-Carlo based sampling technique is used in this paper. This is described briefly in section 3.1 and in more detail in [14]. The original simulation model is run for these samples to obtain the $n$ corresponding values for each of the outputs. These data will be referred to as training data. In general, these training data are standardised (zero mean, unit variance) to overcome influences from parameter units. Each output is then modelled separately or together with a metamodelling technique. With these techniques, the training process results in an independent model to estimate new input/output combinations within the range of the sampled combinations. The more training data are used, the better the metamodel can perform in general. It is however possible that the training data are perfectly fit, while unseen data are not approximated well at all. This phenomenon is 
called overfitting and can be avoided by employing generalisation methods that reduce the complexity of the model by

- regularisation: limiting the Euclidean norm of the coefficients vector, in order to avoid unnecessarily large coefficients, or

- pruning: reducing the number of coefficients before or after fitting them, in order to avoid too many coefficients.

Amongst the variety of readily available algorithms for each of the metamodelling groups described in section 2.3, algorithms employing these generalisation methods are selected. In this paper, all algorithms are provided by MATLAB toolboxes and are referred to when the techniques are described.

Each of the selected algorithms contain several settings that have to be defined by the user. Different settings might result in differing metamodels, of which only the best is retained, selected via a model selection criterion. Such a criterion indicates the trade off between the goodness of fit and model complexity in order to avoid more coefficients than needed. The Akaike information criterion $(A I C)$ [15] is commonly used for that purpose and is given by

$$
A I C=n \log (S S E)+2 n_{p}
$$

with $n$ the number of training samples, $S S E$ the sum of squared errors, and $n_{p}$ the effective number of parameters. The model with the lowest $A I C$ score has the best trade off between the smallest error and the least number of coefficients, the latter of which enhances the generalisation ability of the model.

\subsection{Validation}

The metamodelling techniques that will be described in section 2.3 are thus all optimised to prevent overfitting of the training data, enhancing the goodness of fit on unseen data. To validate this performance however, goodness of fit needs to be assessed on unseen data, the validation data, instead of on training data. Extra input/output combinations are thus created via sampling methods and used to compare the predictions of the metamodel with the original model output.

This goodness of fit can be determined by several indicators. Amongst others, Root Mean Squared Error $(R M S E)$, coefficient of determination $\left(R^{2}\right)$, and Maximal Absolute Error $(M A E)$ are commonly used indicators. They are illustrated in Fig. 1 and given by

$$
\begin{gathered}
R M S E=\sqrt{\frac{1}{n} \sum_{i=1}^{n}\left(\hat{y}_{i}-y_{i}\right)^{2}} \\
R^{2}=1-\frac{\sum_{i=1}^{n}\left(\hat{y}_{i}-y_{i}\right)^{2}}{\sum_{i=1}^{n}\left(\bar{y}-y_{i}\right)^{2}} \\
M A E=\max \left(\left|\hat{y}_{1}-y_{1}\right|, \ldots,\left|\hat{y}_{n}-y_{n}\right|\right)
\end{gathered}
$$

with $y_{i}$ the original model outputs, $\hat{y}_{i}$ the metamodel outputs, $\bar{y}$ the mean simulation output value, and $n$ the number of samples.

$R M S E$ measures the standard deviation of the error between the metamodel and original output. It thus indicates the overall approximation ability of the metamodel; the lower the value, the better the approximation. The coefficient of determination $R^{2}$ is a relative indicator for the overall approximation. In fact, it indicates the correlation between the original model output and the metamodel output. A perfect correlation is given by an $R^{2}$-value of 1 . Complementary to the above-mentioned indicators, $M A E$ indicates the maximal absolute error that can be expected, and is thus an indicator for the local approximation ability of the metamodel. RMSE and $M A E$ can be made relative to the standard deviation of the simulation output in order to allow a comparison between several outputs of varying magnitudes. In this paper, this is however not done since $R^{2}$ already provides such a relative indicator.

Depending on the metamodel's goal, one or more validation indicators are selected. One of the most important steps in metamodelling is then to select validation criteria for these indicators. These criteria depend on the problem as well, and might be hard to determine. As the accuracy required for a predictive metamodel is usually very high, a low $R M S E$ and $M A E$, and a high $R^{2}$ are sought $[2,13]$. 


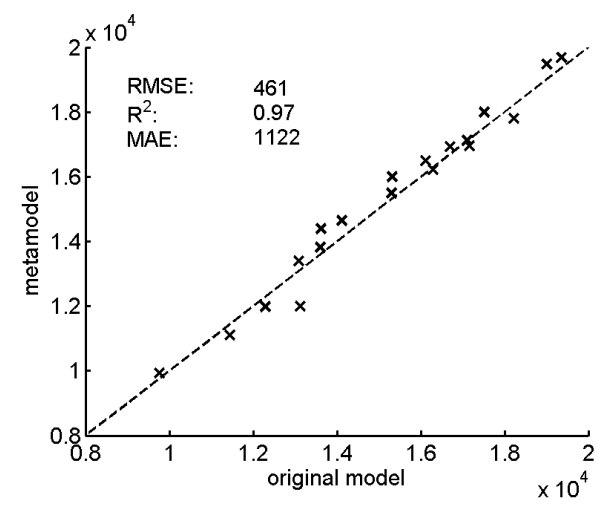

Figure 1: Illustration of goodness-of-fit indicators to compare the metamodel output with the original model output.

\subsection{Metamodelling techniques}

As mentioned in section 2.1, each of the five selected metamodelling techniques uses at least one generalisation method to avoid overfitting, and in each algorithm, several settings have to be defined by the user. Several options are therefore selected and the best model is then selected based on their $A I C$ scores, as summarised in Table 1 .

\subsubsection{Polynomial regression}

$\mathrm{PR}$ is one of the most widely used metamodelling techniques and fits an $m^{\text {th }}$ order polynomial between the sampled input and output data using the method of least squares. In general, the model is a function

Table 1: Overview of algorithms and user-defined settings for the metamodelling techniques.

\begin{tabular}{|c|c|c|c|c|c|}
\hline & $\begin{array}{l}\text { generalisation } \\
\text { method }\end{array}$ & $\begin{array}{l}\text { internal } \\
\text { settings }\end{array}$ & $\begin{array}{l}\text { comparison } \\
\text { criterion }\end{array}$ & external settings & $\begin{array}{l}\text { comparison } \\
\text { criterion }\end{array}$ \\
\hline PR & $\begin{array}{l}\text { regularisation } \\
\text { (ridge regression) }\end{array}$ & & & $\begin{array}{l}\text { - model order }(1,2,3) \\
\text { - regularisation factor }(50 \\
\left.\text { values between } 10^{-6} \text { and } 10^{6}\right)\end{array}$ & $A I C$ \\
\hline MARS & pruning (GCV) & & & $\begin{array}{l}\text { maximal number of basis } \\
\text { functions (values between } \\
20 \text { and } 140 \text { ) }\end{array}$ & $A I C$ \\
\hline KR & pruning (order 0) & & & $\begin{array}{l}\text { correlation function (Gaussian, } \\
\text { exponential, Matérn32 and } \\
\text { Matérn52) }\end{array}$ & $A I C$ \\
\hline RBF & $\begin{array}{l}\text { - pruning } \\
\text { (forward sel. BIC) } \\
\text { - regularisation } \\
\text { (ridge regression) }\end{array}$ & $\begin{array}{l}\text { - scale factor } \\
(10 \text { values } \\
\text { between } 10 \% \\
\text { and } 100 \%) \\
\text { - reg. factor ( } 50 \\
\text { values between } \\
10^{-6} \text { and } 10^{6} \text { ) }\end{array}$ & $B I C$ & $\begin{array}{l}\text { - basis function type (Gaussian, } \\
\text { Cauchy, multiquadric, inverse) } \\
\text { - technique (forward selection, } \\
\text { ridge regression) }\end{array}$ & $A I C$ \\
\hline NN & $\begin{array}{l}\text { regularisation } \\
\text { (Bayesian) }\end{array}$ & & & $\begin{array}{l}\text { - architecture (feed forward, } \\
\text { cascade forward) } \\
\text { - number of layers }(1,2) \\
\text { - number of neurons (between } \\
1 \text { and } 20 \text { ) }\end{array}$ & $A I C$ \\
\hline
\end{tabular}


of the form

$$
\hat{y}=b_{0}+\sum_{n=1}^{m} \sum_{i=1}^{p} b_{n i} x_{i}^{n}+\sum_{n=1}^{m} \sum_{k=1}^{m} \sum_{i=1}^{p} \sum_{j=1}^{p} b_{n k i j} x_{i}^{n} x_{j}^{k}
$$

with $\hat{y}$ the estimated output, $x$ the input vectors, $p$ the number of inputs, $m$ the order of the polynomial, and $b$ the regression coefficients [3].

In the selected algorithm [16], not only are the summed squares of the errors minimised, but also the magnitude of the coefficients to avoid overfitting as described before. Therefore, the least square cost function is modified by an additional term, which aims at keeping the Euclidean norm of the coefficients vector small, often referred to as ridge regression. The cost function is then

$$
\sum(\hat{y}-y)^{2}+\gamma \sum b^{2}
$$

with $\gamma$ the regularisation factor [17].

Several model orders, $m$, and regularisation factors, $\gamma$, can be used to determine the coefficients, $b[16]$. In this paper, model orders one, two, and three, and 50 values for $\gamma$ logarithmically distributed between $10^{-6}$ and $10^{6}$ are chosen to build the polynomial metamodels.

\subsubsection{Multivariate adaptive regression splines}

MARS models are of the form

$$
\hat{y}=\sum_{i=1}^{k} c_{i} B_{i}(x)
$$

with $\hat{y}$ the estimated output, $x$ the input vectors, $k$ the number of basis functions, $B_{i}$, and $c_{i}$ the weight factors $[3,18]$. Non-linearities between outputs and inputs can be taken into account because of the use of hinge functions as basis functions. A hinge function has the form $\max (0, x-$ constant $)$ or $\max (0$, constant $-x)$ and thus produces a kink. The basis functions in Eq. 7 are a constant, a hinge function, or a product of hinge functions to take interactions into account. Both the hinge functions and weight factors have to be determined, which is done through a forward selection and a backward deletion iterative approach. In the forward phase, basis functions giving the largest reduction in the training error are added until (1) the (change in) training error becomes small, (2) more weight factors than training samples are expected, or (3) the user-defined maximum number of basis functions is reached. Typically an overfit model is the result. In the backward phase, the model is pruned by trading off goodness of fit against model complexity using the Generalised Cross-validation $(G C V)$ criterion, similar to $A I C$, given by

$$
G C V=M S E /\left(1-\frac{n_{p}}{n}\right)^{2}
$$

with $M S E$ the mean-squared error. This pruning means that the least effective terms are deleted one by one to improve the generalisation ability [19]. Any value can be selected for the maximum number of basis functions, but the calculation time significantly increases when adding more functions. Several values between 20 and 140 are selected in this paper.

\subsubsection{Kriging}

$\mathrm{KR}$ is an interpolation method originating in the field of geostatistics [20] and was proposed by Sacks et al. [21] for computer experiments without random errors, as a better alternative to statistical techniques designed for physical experiments with random errors, such as PR. KR models provide a global regression model analogous to simple polynomial regression that is augmented with a Gaussian process to interpolate the residuals. A well-specified regression model is thus not needed to obtain a well-performing model, in contradiction to the other techniques explained in his paper. KR models are of the form

$$
\hat{y}=\sum_{i=1}^{k} b_{i} h_{i}(x)+Z(x)
$$


with $\hat{y}$ the estimated output, $x$ the input vectors, $k$ the number of polynomial basis functions, $h_{i}, b_{i}$ the regression coefficients determined with the least squares method, and $Z$ a Gaussian process. This process has mean zero, variance $\sigma^{2}$, and correlation functions $\psi\left(x, x^{\prime}\right)$, between any two samples of the input vector. A typical correlation function is the Gaussian:

$$
\psi\left(x, x^{\prime}\right)=\exp \left(-\sum_{i=1}^{p} \theta_{i}\left|x_{i}-x_{i}^{\prime}\right|^{2}\right)
$$

Several correlation function types are available, which are all determined by a correlation parameter, $\theta$, with the same dimension as the input vector, i.e. $p$. These correlation functions inform nearby sample points about the residuals in these points. The smaller the distance between the sample points, the more the prediction of one of the points is influenced by the other. Selecting satisfying correlation functions and correlation parameter values is thus crucial for this method. The former have to be selected by the user, while the latter are automatically determined by maximum likelihood estimation $[1,22,23]$. Note that when the training data are noisy, stochastic kriging can be used, which combines interpolation and smoothing to avoid overfitting [23]. The considered data is noisy when two simulations with the same input does not result in the same output.

Four correlation functions (Gaussian, exponential, Matérn32 and Matérn52) [22] are selected in this paper. Note that in kriging, more coefficients than available samples cannot be estimated, but due to the Gaussian process, a well-performing model can be obtained with low-order polynomials. Therefore, a model order of zero is chosen for all models.

\subsubsection{Radial basis function network}

$\mathrm{RBF}$ networkss are of the form

$$
\hat{y}=\sum_{i=1}^{k} w_{i} h_{i}(x)
$$

with $\hat{y}$ the estimated output, $x$ the input vectors, $k$ the number of basis functions $h_{i}$, and $w_{i}$ the weights. A typical Gaussian basis function is of the form

$$
h_{i}(x)=\exp \left(-\frac{\left\|x-c_{i}\right\|^{2}}{r_{i}^{2}}\right)
$$

with $c_{i}$ the centre and $r_{i}$ the radius of basis functions, $h_{i}[24]$. Other similar basis functions are used as well, like the Cauchy, the multiquadric, and the inverse function. Both weights, $w_{i}$, and basis function parameters, $c_{i}$ and $r_{i}$, have to be determined. The centres are usually taken to be equal to the input matrix $X$. Each basis function centre is thus $p$-dimensional and as many basis functions as initial samples are created. In general, for each dimension, the radii are chosen to be equal to the span of the training set inputs, and are thus the same for each basis function. However, it is preferable to apply a scale factor $(\leq 100 \%)$ to this radius to avoid underfitting, as otherwise the RBFs will probably be too wide. A set of scale factors can be provided by the user and the best scale is then selected in the algorithm based on the Bayesian Information Criterion $(B I C)$ score. $B I C$ is very similar to $A I C$ and is calculated with

$$
B I C=n \log (S S E)+n_{p} \log (n)
$$

Either forward selection or ridge regression can be performed to select some of the available basis functions (including a bias unit). If all basis functions are selected, RBF is seen as an interpolation technique. Forward selection compares models made up of different subsets of basis functions. Basis functions that best reduce the sum-squared error are added one by one, until the $B I C$ score stops decreasing to avoid models that are too complex. Weight factors are determined based on the sum-squared error as well. Ridge regression selects all available basis functions and augments the sum-squared error with an extra term penalising large weights to avoid overfitting analogous to polynomial regression. Several regularisation factor values have to be provided by the user and the best one is then internally selected based on the BIC scores [25]. Ten scale 
factors between $10 \%$ and $100 \%$ and 50 regularisation factors logarithmically distributed between $10^{-6}$ and $10^{6}$ are chosen in order to automatically determine an optimum. The four basis function types are used to create networks with both generalisation techniques.

\subsubsection{Sigmoidal transfer function network}

Sigmoidal transfer function networks are standard neural networks (NN). Neural networks consist of a first layer with input neurons, a final layer with output neurons, and any number of hidden layers in between, as illustrated in Fig. 2a [26]. The neurons are transfer functions and in this paper they are sigmoidal in all layers except the final layer, in which they are linear. In particular, tan-sigmoidal functions are selected as illustrated in Fig. 2b. A single feed forward neural network with one output is constructed with the following equations:

$$
\begin{gathered}
\eta_{j}=\sum_{i=1}^{p} w_{x i} x_{i}+\beta_{x j} \\
f\left(\eta_{j}\right)=\frac{2}{1+\exp \left(-2 \eta_{j}\right)}-1 \\
\hat{y}=\sum_{j=1}^{m} w_{y j} f\left(\eta_{j}\right)+\beta_{y j}
\end{gathered}
$$

Here, a weighted, sum $\eta_{j}$, of the $p$ input parameter values, $x_{i}$, with input weights, $w_{x i}$, and a bias value, $\beta_{x j}$ (Eq. 14) feeds forward to $m$ tan-sigmoidal transfer functions $f\left(\eta_{j}\right)$ (Eq. 15). The $m$ outputs of the hidden layer are then linearly combined with a bias, $\beta_{y j}$, and weights, $w_{y j}$, into the estimated output, $\hat{y}$ (Eq. 16) [1]. When more hidden layers are available, the outputs of the previous hidden layer are considered as inputs and Eq. 14 and 15 are repeated. The outputs of the final hidden layer are then linearly combined into $\hat{y}$. A cascade forward construction is also considered, in which connections between non-adjacent layers are possible. Biases and weights are trained by least-squares minimisation, which is modified for regularisation similar to Eq. 6 to improve generalisation. For that purpose, Bayesian regularisation is applied, which uses an iterative approach to determine weights, biases and the regularisation factor by considering them as random variables [27-29]. Bayesian regularisation should not be confused with $B I C$.

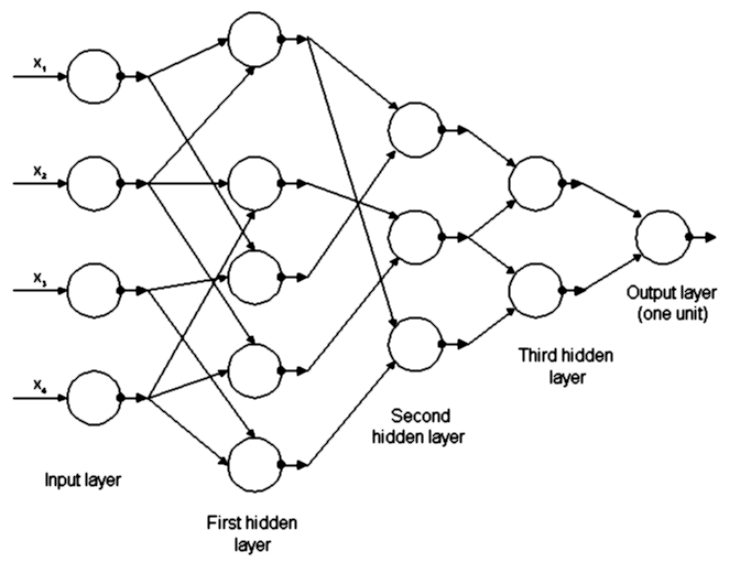

(a)

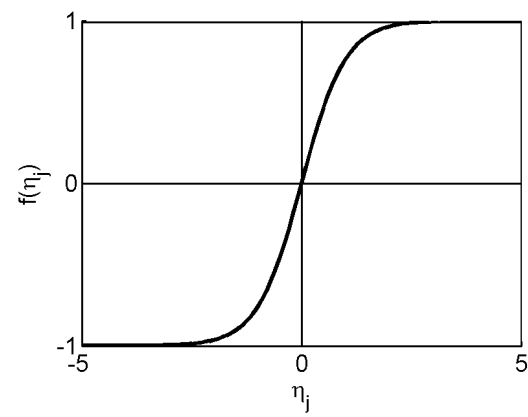

(b)

Figure 2: Feedforward neural network with three hidden layers (a) and tan-sigmoid transfer function (b).

Creating a well-performing neural network depends on the choice for the number of layers and neurons in the network [30]. Therefore, one or two hidden layers each with up to 20 neurons and both feed forward and cascade forward constructions are used to build sigmoidal neural networks [31]. 


\section{Metamodelling strategy}

A metamodelling strategy independent of the metamodel type is proposed in which the metamodels are both fitted and validated while the number of simulation runs is constrained. Since the sampling strategy used to fit the metamodel might be of importance, this topic is handled first.

\subsection{Sampling strategy}

Several studies have highlighted the importance of selecting a well-performing sampling scheme for training and validation $[4,32,33]$. Random sampling, Latin Hypercube schemes, space-filling sampling such as optimised Latin Hypercubes, orthogonal arrays and quasi-Monte Carlo methods, and also classic schemes such as (fractional) factorial, central composite and optimal schemes have been explored for metamodelling. Simpson et al. [1] state a consensus on the best-performing scheme across several problems is only reached for space-filling sampling schemes. Such schemes are thus preferred and will be used in this paper. More information on space-filling schemes can be found in [14].

\subsection{Fitting and validation strategy}

Section 2 described both fitting and validation theory using training and validation samples respectively. Previous research [34] illustrated that different sample sets might however result in different validation indicator values. To avoid getting good or bad values by coincidence, it is recommended to train and validate the metamodel for as many samples as possible. This set of indicator values can then be evaluated on minimal, average and maximal values in order to reliably evaluate the created metamodel.

To balance between time efficiency and reliability however, a new metamodelling strategy as proposed by Van Gelder et al. [12] is employed (Fig. 3). A metamodel is first trained on one small sample set according to one of the techniques in section 2.3 while seeking the best user-defined settings. Then an additional sample set is created and a $k$-fold cross-validation is performed using the optimal settings to check the reliability of validation indicator values. This implies that of the $k$ available sample sets, one set is used as a validation set, while the other $k-1$ sets are training sets. This is repeated $k$ times with each of the $k$ sample sets used exactly once as a validation set, resulting in $k$ validation indicator values [2]. Sample sets are systematically added until the minimal, average and maximal values of selected validation indicators converge to the desired validation criteria. The reliability of the metamodel can be judged on the spread of these indicator values.

In this strategy, users can select a metamodelling technique of their choice. Hence, if the built metamodel remains insufficiently reliable, another metamodelling technique can be selected to obtain a potentially better reliability of the validation indicators.

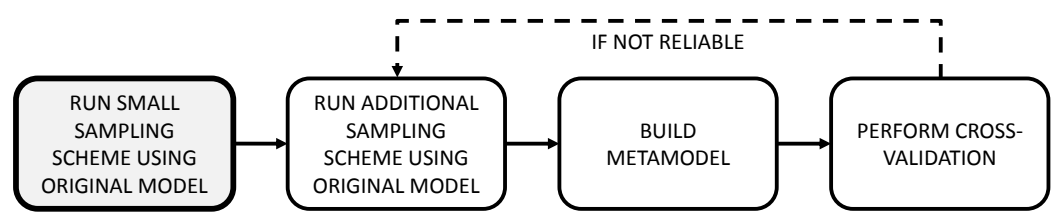

Figure 3: Flowchart of metamodelling strategy.

\section{Application example}

The described metamodelling strategy is illustrated using a building energy simulation (BES) example. When performing a robust optimisation of energy demand and thermal comfort in order to select design variables of a specific dwelling as illustrated in [12], the calculation time of a dynamic BES model can be a barrier. The construction of metamodels can greatly facilitate this, because of their highly reduced calculation time. Therefore, this application example aims at constructing a reliable metamodel to replace the time-consuming original BES model. 


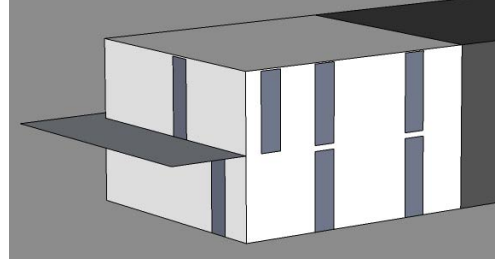

(a) North facade

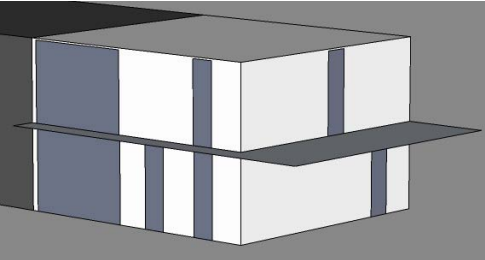

(b) South facade

Figure 4: Case study - semi-detached house.

\subsection{Building model}

A semi-detached dwelling (see Fig. 4) with a floor area of $140 \mathrm{~m}^{2}$, overhangs for sun shading, and an uninsulated, ventilated basement [35] is modelled in the Integrated District Energy Assessment Simulation (IDEAS) tool in Modelica, developed by Baetens et al. [36]. The dwelling is modelled as consisting of two main zones to differentiate between areas typically inhabited during the day and night. The adjacent dwelling is considered to be at a constant temperature of $19{ }^{\circ} \mathrm{C}$ and the typical moderate climate year of Uccle (Belgium) is used as the outdoor climate. An occupancy profile of a full-time working couple is assumed. An ideal heating system coupled to this occupancy profile and an exhaust ventilation system are incorporated in the model. To optimise the summer comfort, additional summer ventilation is taken into account: when occupants are present and the day zone temperature exceeds the user-dependent comfort temperature, the air change rate is doubled for the next six hours or until the occupants leave the dwelling. This algorithm simulates the user behaviour to help achieve a comfortable indoor climate. The model outputs that we are interested in are the heat demand and the number of hours with temperatures exceeding $25^{\circ} \mathrm{C}(\mathrm{TE} 25)$.

\subsection{Inputs}

Table 2 lists the probabilistic inputs and ascribed distributions for which metamodels need to be constructed. Some inputs have strictly discrete values, while others are described by continuous uniform, normal or Weibull distributions. These distributions are all based on the values that can be expected for newly built dwellings in Belgium [37].

\subsection{Sampling scheme}

Following Simpson et al. [1], a space-filling sampling scheme is chosen in this paper to create training and validation samples: a maximin Latin Hypercube sampling scheme. This is a distance-based scheme that maximises the minimal distance between Latin Hypercube sampling points [38]. All inputs in Table 2 are sampled according to their probability distributions. To allow several different sample sizes for model training and cross-validation, 25 sets of 20 maximin samples are created and run with the original model, therefore giving 500 samples in total.

\subsection{Metamodels}

Since both reliability and calculation efficiency are crucial in metamodelling, techniques with good approximation ability using only a few samples are preferable, however more samples usually increases the goodness of fit. Therefore, training sets with several different samples sizes are used to construct metamodels for heat demand and TE25 with the techniques described in section 2.3. The models based on 500 training samples can be considered as the best possible metamodels. In order to make the metamodels physically relevant, the metamodel outputs for heat demand and TE25 values are forced to be strictly positive. As described before, the best settings for each technique and sample size are chosen based on $A I C$ scores, resulting in one metamodel per technique per training sample size.

The goodness of the fit of these metamodels is first validated on 20 unseen samples according to section 2.2. This will provide insight into the approximation ability of the techniques and the influence of the sample size in order to mutually compare them. The best settings per technique and per sample size are 
Table 2: Probabilistic input parameters.

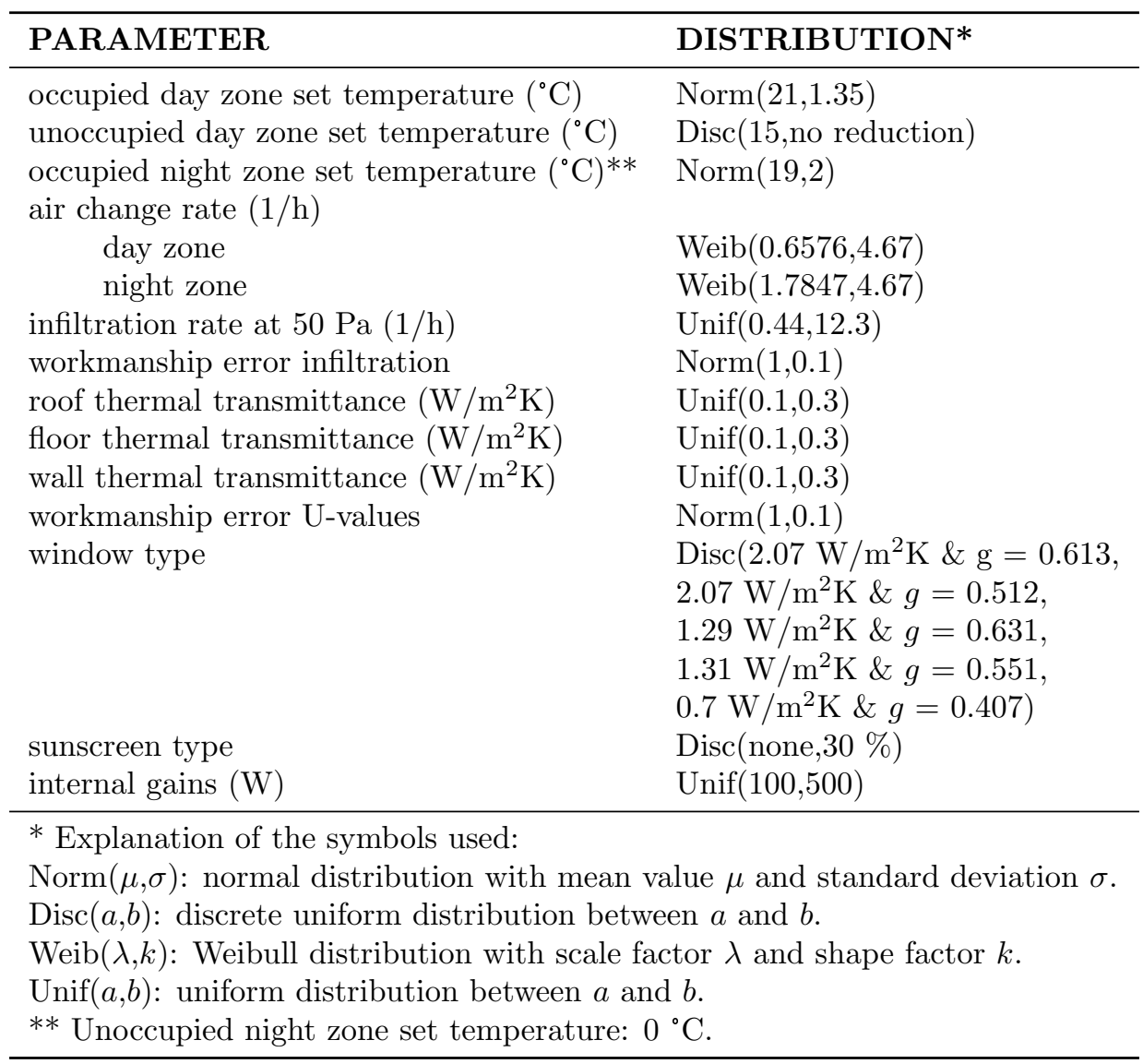

furthermore used in the $k$-fold cross-validation described in section 3.2, performed for $k$ sample sets varying between two and ten. This cross-validation allows the examination of the reliability of the metamodelling techniques.

\section{Results}

\subsection{Setting comparison}

As mentioned earlier, several settings have to be defined and selected by the user for each of the metamodelling techniques. In this paper, only the best metamodel for each technique is retained, based on the AIC model comparison criterion. The lower this value, the better the generalisation ability of the model. Table 3 therefore compares the best and worst settings in view of this $A I C$ score for metamodels trained on 100 samples. One can see that the settings have larger influence on $A I C$ scores for PR, RBF and NN than for MARS and KR. MARS and KR are thus more robust.

Note that the best and worst settings in Table 3 are only valid for the considered case study and training sample size. Other problems and sample sizes might result in other optimal settings. Therefore, it is needed to always test several settings as proposed in this paper. Furthermore, a comparison of $A I C$ scores of several techniques is not meaningful since model structures are totally different.

\subsection{Calculation time}

Table 4 presents a brief comparison of calculation times. These calculation times are computed by timing the training and running process of both outputs, multiple samples, and multiple settings. These times are 
Table 3: Best and worst user-defined setting combinations in view of $A I C$ for metamodels trained on 100 samples.

\begin{tabular}{|c|c|c|c|c|c|}
\hline & & best setting combination & AIC & worst setting combination & AIC \\
\hline $\mathbf{P R}$ & heat demand & $\begin{array}{l}\text { - model order: } 3 \\
\text { - regularisation factor: } 10^{-6} \\
\text { - model order: } 3 \\
\text { - regularisation factor: } 10^{-6}\end{array}$ & $\begin{array}{l}-3512 \\
-3467\end{array}$ & $\begin{array}{l}\text { - model order: } 1 \\
\text { - regularisation factor: } 10^{6} \\
\text { - model order: } 2 \\
\text { - regularisation factor: } 10^{6}\end{array}$ & 459 \\
\hline MARS & $\begin{array}{r}\text { heat demand } \\
\text { TE25 }\end{array}$ & $\begin{array}{l}\max . \text { \# basis functions: } 50 \\
\text { max. \# basis functions: } 40\end{array}$ & $\begin{array}{l}136 \\
133\end{array}$ & $\begin{array}{l}\max . \# \text { basis functions: } 20 \\
\text { max. \# basis functions: } 20\end{array}$ & $\begin{array}{l}215 \\
246\end{array}$ \\
\hline $\mathbf{K R}$ & $\begin{array}{r}\text { heat demand } \\
\text { TE25 }\end{array}$ & $\begin{array}{l}\text { corr. function: Matérn32 } \\
\text { corr. function: Matérn32 }\end{array}$ & $\begin{array}{l}-5739 \\
-5698\end{array}$ & $\begin{array}{l}\text { corr. function: Gaussian } \\
\text { corr. function: Gaussian }\end{array}$ & $\begin{array}{l}-5532 \\
-5635\end{array}$ \\
\hline $\mathbf{R B F}$ & $\begin{array}{r}\text { heat demand } \\
\text { TE25 }\end{array}$ & $\begin{array}{l}\text { - basis func. type: Gaussian } \\
\text { - technique: ridge regression } \\
\text { - basis func. type: Gaussian } \\
\text { - technique: ridge regression }\end{array}$ & $\begin{array}{l}-5941 \\
-5891\end{array}$ & $\begin{array}{l}\text { - basis func. type: inverse } \\
\text { - technique: forward selection } \\
\text { - basis func. type: inverse } \\
\text { - technique: forward selection }\end{array}$ & $\begin{array}{l}-3468 \\
-3393\end{array}$ \\
\hline NN & heat demand & $\begin{array}{l}\text { - architecture: feed forward } \\
\text { - number of layers: } 2 \\
\text { - number of neurons: } 17,19 \\
\text { - architecture: feed forward } \\
\text { - number of layers: } 1 \\
\text { - number of neurons: } 9\end{array}$ & -2983 & $\begin{array}{l}\text { - architecture: feed forward } \\
\text { - number of layers: } 2 \\
\text { - number of neurons: } 16,1 \\
\text { - architecture: feed forward } \\
\text { - number of layers: } 2 \\
\text { - number of neurons: } 1,16\end{array}$ & 461 \\
\hline
\end{tabular}

Table 4: Indicative calculation time for one output and one set of model settings.

\begin{tabular}{lrrrrr}
\hline & PR & KR & MARS & RBF & NN \\
\hline training time 100 samples & $2 \mathrm{~s}$ & $4 \mathrm{~s}$ & $3 \mathrm{~s}$ & $2 \mathrm{~s}$ & $40 \mathrm{~s}$ \\
running time 100 samples & $0.2 \mathrm{~s}$ & $0.08 \mathrm{~s}$ & $0.03 \mathrm{~s}$ & $0.02 \mathrm{~s}$ & $0.1 \mathrm{~s}$ \\
\hline reference running time for 1 & \multicolumn{5}{l}{ simulation in original model: $500 \mathrm{~s}$} \\
\hline
\end{tabular}

then averaged to obtain the time indicators of Table 4.

First of all, one can notice a huge reduction in calculation time once a metamodel is constructed. All metamodelling techniques result in very time-efficient models, however the calculation times still differ.

More differences can be found in training times. Training one single output with one collection of model settings on 100 training samples is also very time-efficient. However, variation can be found in these single training times; major differences are due to the number of potential model settings as seen in Table 1 . While for MARS models only a set of maximal number of basis functions need to be tested, NN require a comparison of numerous network architectures and layers. Furthermore, following Table 3, these NN settings seem to be more dominant in obtaining well-performing models than the maximal number of basis functions

Note that the time needed to construct the metamodels of computational-expensive models is usually far below the time needed to get the training data, even when numerous settings have to be compared. As the computation time of the original model can be easily reduced by parallel computing, the training time might be important for less expensive models such as the BES model in this example. As stated by Mullur and Messac [7], a slightly longer calculation time is usually tolerated when this is improving accuracy.

\subsection{Validation}

Fig. 5 compares metamodel outputs and original outputs of the validation set for models trained on 20, 200, and 500 samples. It illustrates that adding more training samples results in better metamodels. Fig. 5 also shows that some outputs are more difficult to reproduce with a metamodel than others. For heat 
demand, KR and NN can already provide well-approximating models with only 20 training samples, while not for TE25. All applied techniques appear to be suitable to create metamodels of heat demand, while NN seems best for TE25.

The reliability of validation indicators as a function of the number of sample sets is illustrated in Fig. 6 . As described earlier, all sets are used once as a validation set while the remaining sets are used as training sets. Minimum, average, and maximum values of the indicators for a given sample size indicate how sensitive the values are to training and validation samples. The better the mean value is and the closer the minimum and maximum are to each other, the more reliable the constructed model. Again, one can see that adding more training samples generally results in better metamodels and that some outputs are more difficult to model than others. From this perspective, KR and NN are most reliable for heat demand, however when more samples are used, MARS also performs well. For TE25, it seems to be much harder to have very accurate metamodels: only $\mathrm{KR}$ and $\mathrm{NN}$ seem to match the original model and many more training samples are needed to obtain similar indicator values. Therefore particular attention needs to be paid to the construction of a metamodel TE25 when a high accuracy is desired.

\section{Discussion and conclusions}

This paper proposed a metamodelling strategy dealing with both efficiency and reliability. Moreover, it provided an overview of five metamodelling techniques, all optimised in order to avoid overfitting: PR, MARS, KR, RBF, and NN. These techniques were compared by applying them on a BES example. All models were created based on a set of input/output combinations, the training set, with up to 25 sets of 20 samples. For each technique and each sample size, several settings were tested of which the best were selected based on the value of $A I C$, representing a trade off between the goodness of fit and model complexity. To assess the quality of the developed metamodels, a set of 20 validation samples was used and a k-fold cross-validation was performed.

\subsection{Reliable metamodelling strategy}

Usually, the more training samples available, the better the original model was approximated by the metamodel. Unfortunately, it is not always possible to create as many samples as desired due to calculation time. For that reason, it is important to examine how accurate the metamodel has to be. However, this is dependent on the goal of the model: predictive models usually need to be highly accurate. The accuracy should be checked on validation data not used in the training and the metamodel should only be applied within the range of the training data values. It should be mentioned that the accuracy of the metamodel is at least as important as the calculation time: as long as we need less initial samples for the metamodel than we should need for an analysis on the original model, we can expect that time will be saved.

In order to construct a reliable metamodel with as few simulation sets as needed, following Van Gelder et al. [12], a metamodelling strategy was proposed. Because training and validation sets may be of influence for the validation of the model, cross-validation is preferred. Sample sets are systematically added to construct a metamodel meeting the validation criteria. In this paper it is shown that for some metamodels, reliable performance is obtained with a small sample size, while for other models, more samples were needed.

\subsection{Metamodelling techniques comparison}

Depending on non-linearity, the dimension, and noisiness of the initial model, other techniques can provide better models [3]. The overall best-performing metamodelling technique for this building energy example was NN and PR performed worst. Well-performing models could however be obtained with all techniques. In general, MARS and RBF needed more training samples to obtain the same accuracy compared to KR and NN. This confirms the findings in literature as summarised in the introduction [1,3-8]. When dealing with a very time-consuming original model, $\mathrm{KR}$ and $\mathrm{NN}$ are thus preferred because less training data would be needed. Of these two, KR has a far lower training time, however the models are much harder to interpret. When comparing the metamodelling techniques in usability, MARS is preferred by the authors to KR and NN because of its simplicity and clear relationship between inputs and outputs. Compared to other 
techniques, fewer settings with less influence have to be chosen by the user. MARS can thus be considered as more robust.

It should also be noted that preferences are dependent on the selected algorithms. Further development of these algorithms might thus result in other preferred techniques.

\section{Acknowledgements}

This work was mainly performed under the framework of IEA EBC Annex 55 Subtask 2 [39]. The authors are thankful to all participants for the fruitful discussions during these meetings. They would also like to thank Philippe Dreesen from the Department of Fundamental Electricity and Instrumentation at Vrije Universiteit Brussel for helping to understand and implement some metamodelling principles. The proof readers and reviewers of this paper are moreover gratefully acknowledged.

\section{References}

[1] T. W. Simpson, J. D. Peplinski, P. N. Koch, J. K. Allen, Metamodels for computer-based engineering design : survey and recommendations, Engineering with Computers 17 (2001) 129-150.

[2] G. G. Wang, S. Shan, Review of Metamodeling Techniques in Support of Engineering Design Optimization, Journal of Mechanical Design 129 (4) (2007) 370.

[3] R. Jin, W. Chen, T. W. Simpson, Comparative studies of metamodelling techniques under multiple modelling criteria, Structural and Multidisciplinary Optimisation 23 (2001) 1-13.

[4] M. F. Hussain, R. R. Barton, S. B. Joshi, Metamodeling: Radial basis functions, versus polynomials, European Journal of Operational Research 138 (1) (2002) 142-154.

[5] R. Jin, X. Du, W. Chen, The use of metamodeling techniques for optimization under uncertainty, Structural and Multidisciplinary Optimization 25 (2003) 99-116.

[6] H. Fang, M. Rais-Rohani, Z. Liu, M. Horstemeyer, A comparative study of metamodeling methods for multiobjective crashworthiness optimization, Computers \& Structures 83 (25-26) (2005) 2121-2136.

[7] A. A. Mullur, A. Messac, Metamodeling using extended radial basis functions: a comparative approach, Engineering with Computers 21 (3) (2006) 203-217.

[8] V. C. Chen, K.-L. Tsui, R. R. Barton, M. Meckesheimer, A review on design, modeling and applications of computer experiments, IIE Transactions 38 (4) (2006) 273-291.

[9] B. Eisenhower, Z. O'Neill, S. Narayanan, V. A. Fonoberov, I. Mezić, A methodology for meta-model based optimization in building energy models, Energy and Buildings 47 (2012) 292-301.

[10] P. M. Ferreira, A. E. Ruano, S. Silva, E. Z. E. Conceic, Neural networks based predictive control for thermal comfort and energy savings in public buildings, Energy and Buildings 55 (2012) 238-251.

[11] C. J. Hopfe, M. T. M. Emmerich, R. Marijt, J. Hensen, Robust multi-criteria design optimisation in building design, in: First Building Simulation and Optimization Conference, 10-11 September, Loughborough, UK, 19-26, 2012.

[12] L. Van Gelder, H. Janssen, S. Roels, Probabilistic design and analysis of building performances: methodology and application example, Energy and Buildings 79 (2014) 202-211.

[13] J. P. Kleijnen, R. G. Sargent, A methodology for fitting and validating metamodels in simulation, European Journal Of Operational Research 120 (2000) 14-29.

[14] H. Janssen, Monte-Carlo based uncertainty analysis: Sampling efficiency and sampling convergence, Reliability Engineering \& System Safety 109 (2013) 123-132.

[15] L. Ljung, System Identification: Theory for the user, Upper Saddle River, NJ, Prentice-Hal PTR, second edition edn., 1999.

[16] MathWorks, Ridge regression, URL http://www.mathworks.nl/help/stats/ridge.html, last consulted on 2014-04-29, 2014.

[17] D. W. Marquardt, R. D. Snee, Ridge Regression in Practice, The American Statistician 29 (1) (1975) 3-20.

[18] J. H. Friedman, Multivariate adaptive regression splines, The Annals of Statistics 19 (1) (1991) $1-141$.

[19] G. Jekabsons, ARESLab: Adaptive Regression Splines toolbox for Matlab/Octave, URL http://www.cs.rtu.lv/jekabsons/regression.html, 2011.

[20] G. Matheron, Principles of geostatistics, Economic Geology 58 (1963) 1246-1266.

[21] J. Sacks, W. J. Welch, T. J. Mitchell, H. P. Wynn, Design and Analysis of Computer Experiments, Statistical Science 4 (4) (1989) 409-423.

[22] I. Couckuyt, a. Forrester, D. Gorissen, F. De Turck, T. Dhaene, Blind Kriging: Implementation and performance analysis, Advances in Engineering Software 49 (3) (2012) 1-13.

[23] J. Staum, Better simulation metamodeling: The why, what, and how of stochastic kriging, Proceedings of the 2009 Winter Simulation Conference (WSC) (2009) 119-133

[24] M. J. L. Orr, Introduction to Radial Basis Function Networks, 1996.

[25] M. J. L. Orr, Matlab functions for Radial Basis Function Networks, URL http://www.anc.ed.ac.uk/rbf/rbf .html, 1999. 
[26] I. Galkin, U. Lowell, Polynomial Neural Networks, URL http://ulcar.uml.edu/ iag/CS/Polynomial-NN.html, last consulted on 2014-04-2, 2014

[27] MathWorks, Improve Neural Network Generalization and Avoid Overfitting, URL http://www.mathworks.nl/help/nnet/ug/improve-neural- network-generalization-and-avoid-overfitting.html, last consulted on 2014-04-28, 2014.

[28] F. D. Foresee, M. T. Hagan, Gauss-Newton approximation to Bayesian regularization, in: Proceedings of the 1997 International Joint Conference on Neural Networks, 1930-1935, 1997.

[29] D. J. C. MacKay, Bayesian Interpolation, Neural Computation 4 (3) (1992) 415-447.

[30] P. Das, C. Shrubsole, B. Jones, I. Hamilton, Z. Chalabi, M. Davies, A. Mavrogianni, J. Taylor, Using probabilistic sampling-based sensitivity analyses for indoor air quality modelling, Building and Environment 78 (2014) 171-182.

[31] MathWorks, Neural Network Toolbox, URL http://www.mathworks.nl/products/neural-network/, last consulted on 2014-04-29, 2014

[32] F. M. Alam, K. R. McNaught, T. J. Ringrose, A comparison of experimental designs in the development of a neural network simulation metamodel, Simulation Modelling Practice and Theory 12 (7-8) (2004) 559-578.

[33] R. T. Johnson, D. C. Montgomery, B. Jones, P. A. Parker, Comparing computer experiments for fitting high-order polynomial metamodels, Journal of Quality Technology 42 (1) (2010) 86-102.

[34] L. Van Gelder, H. Janssen, S. Roels, Metamodelling in robust low-energy dwelling design, in: A. Mahdavi, B. Martens (Eds.), 2nd Central European Symposium on Building Physics, September 9-11, Vienna, Austria, 93-99, 2013.

[35] G. Verbeeck, L. Staepels, S. Roels, L. Van Gelder, G. Bauwens, A.-H. Deconinck, BEP2020 : betrouwbare energieprestaties van woningen - Naar een robuuste en gebruikersonafhankelijke performantie, Tech. Rep. IWT TETRA 110189, URL bep2020.pxl.be, 2013

[36] R. Baetens, R. De Coninck, J. Van Roy, B. Verbruggen, J. Driesen, L. Helsen, D. Saelens, Assessing electrical bottlenecks at feeder level for residential net zero-energy buildings by integrated system simulation, Applied Energy 96 (2012) 74-83.

[37] L. Staepels, G. Verbeeck, S. Roels, L. Van Gelder, G. Bauwens, Evaluation of Indoor Climate in Low Energy Houses, in: Symposium on Simulation for Architecture and Urban Design, April 7-10, San Diego, USA Evaluation, 2013.

[38] R. Jin, W. Chen, A. Sudjianto, An efficient algorithm for constructing optimal design of computer experiments, Journal of Statistical Planning and Inference 134 (1) (2005) 268-287.

[39] H. Janssen, S. Roels, L. Van Gelder, P. Das, IEA EBC Annex 55, Subtask 2, Probabilistic tools, 2014. 


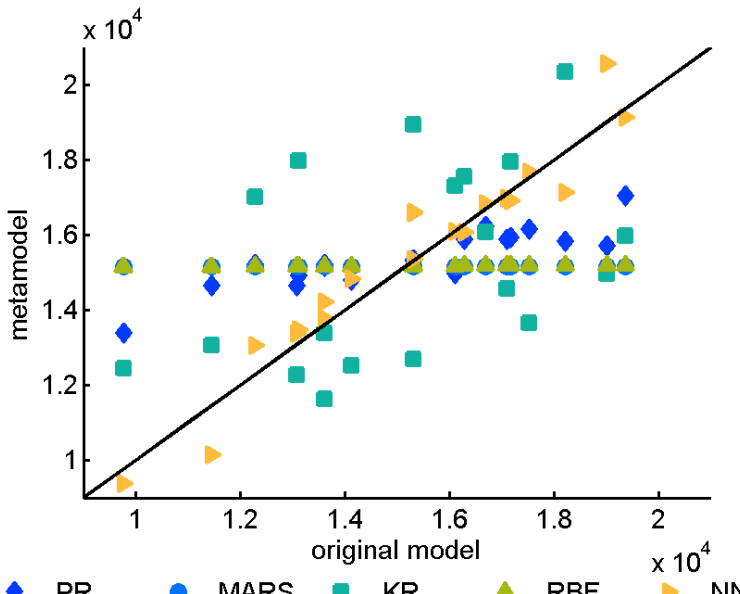

(a) heat demand - 20

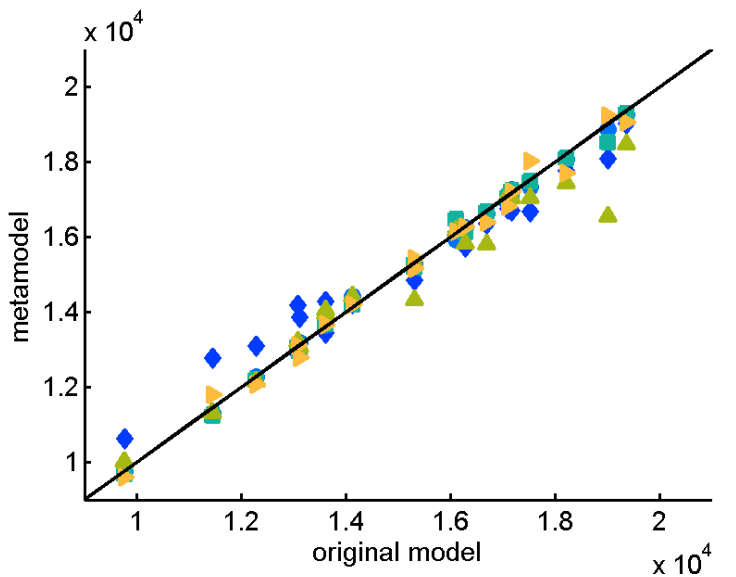

- PR

(c) heat demand - 200

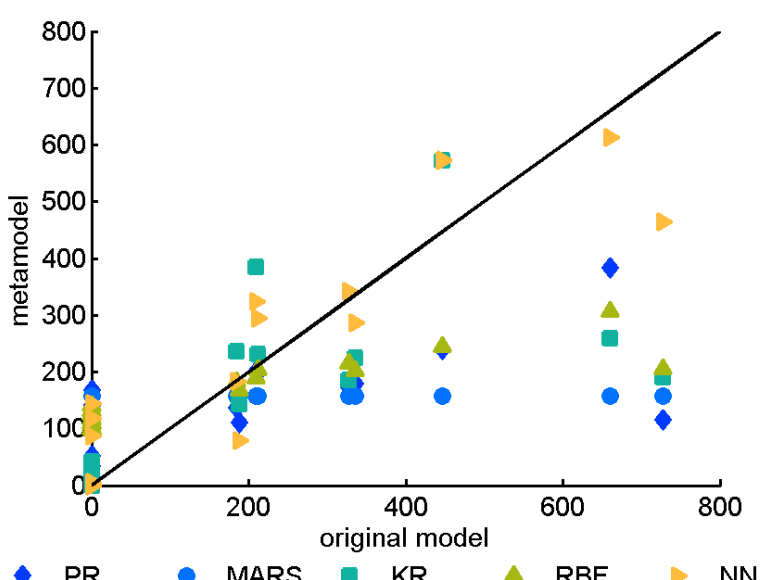

(b) TE25 - 20

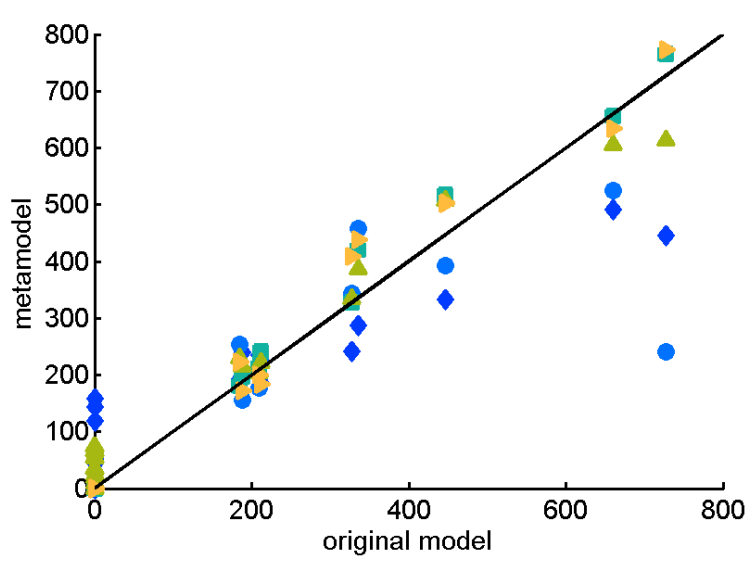

(d) TE25 - 200

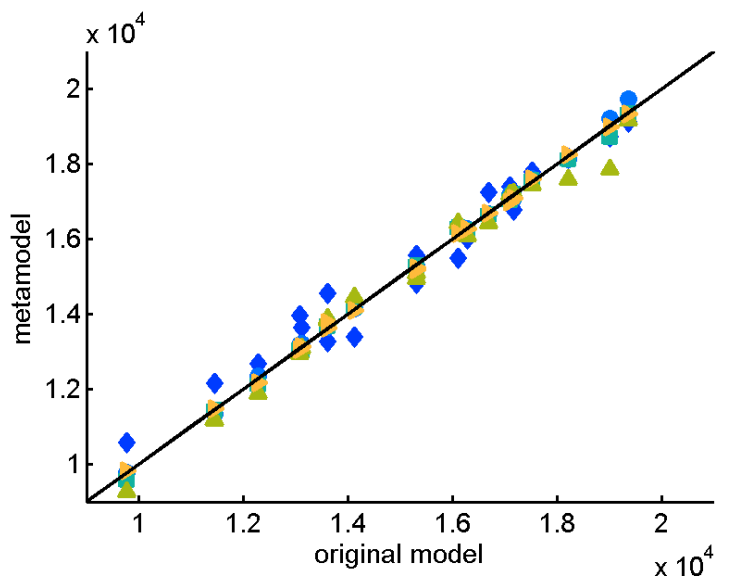

- PR - MARs - KR $\triangle \mathrm{RBF} \gg \mathrm{NN}$

(e) heat demand 500

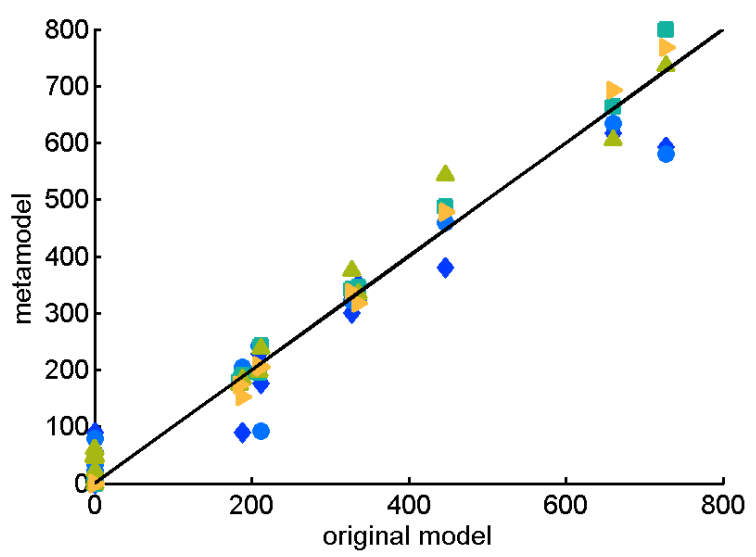

PR

- MARS $\mathrm{KR}$

(f) TE25 - 500

Figure 5: Comparison of original model output with metamodels trained on 20, 200 and 500 samples. 

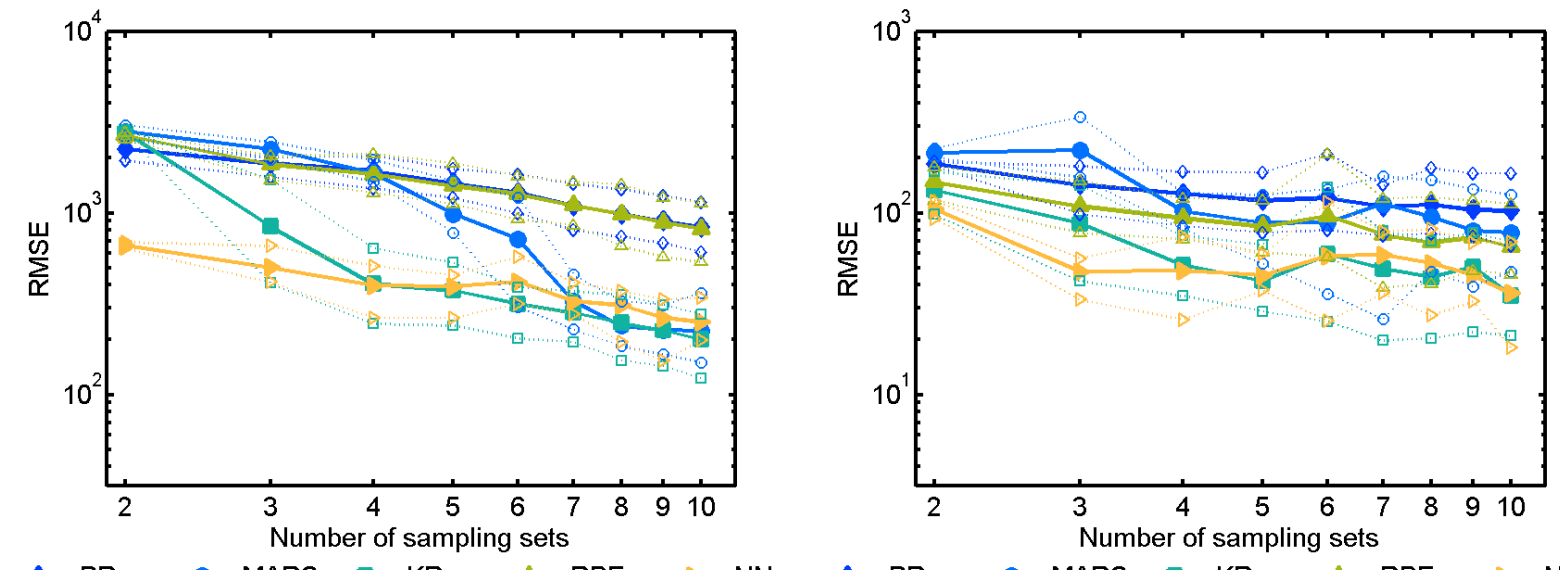

(a) heat demand

(b) TE25
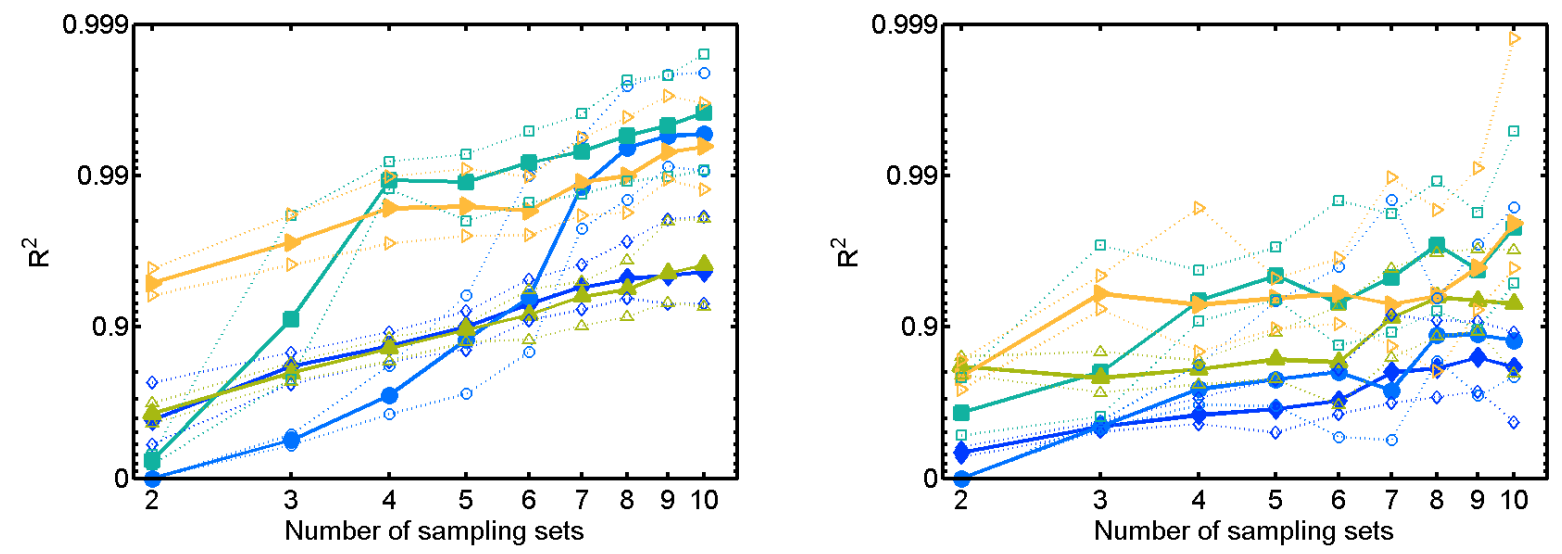

$\rightarrow \mathrm{PR}-\mathrm{MARS}-\mathrm{KR}$
(c) heat demand

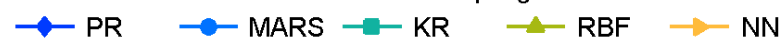

(c) heat demand
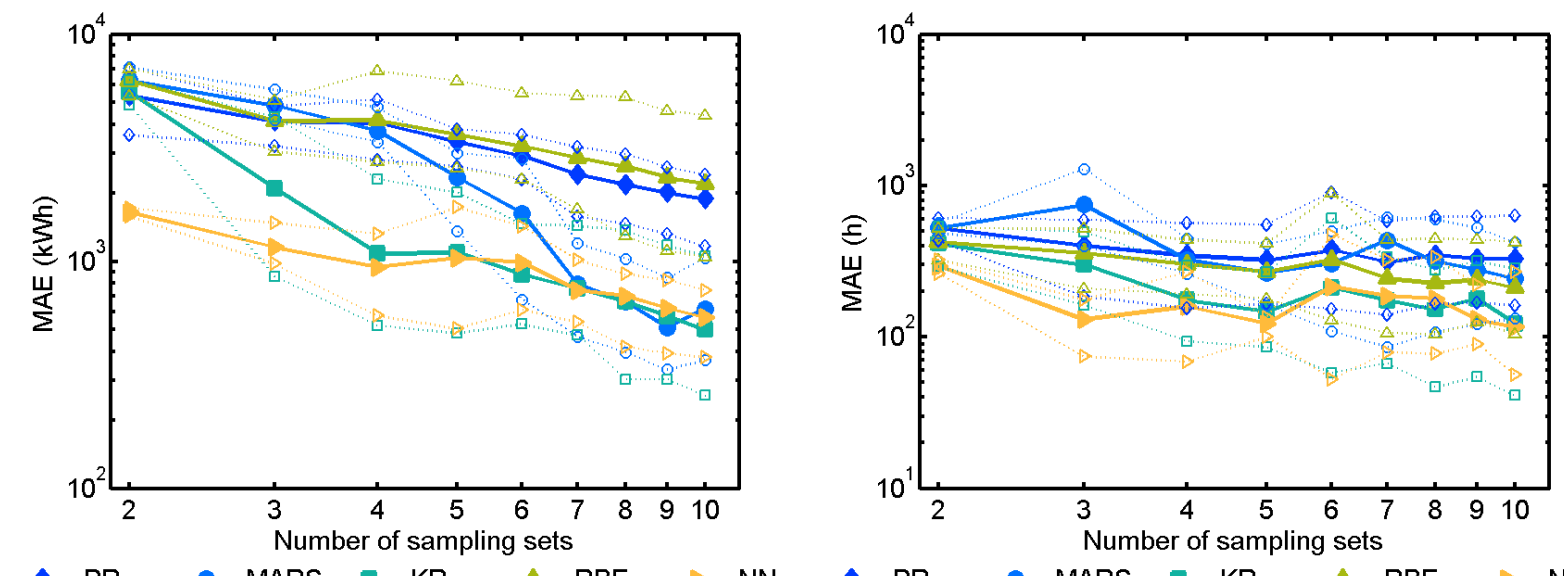

(e) heat demand

(f) TE25

Figure 6: Reliability of validation indicators as a function of the number of training samples for heat demand and TE25 output. Mean values as well as minima and maxima are shown for all techniques. 